\title{
Variant rs2200733 and rs10033464 on chromosome 4q25 are associated with increased risk of atrial fibrillation after catheter ablation: Evidence from a meta-analysis
}

\author{
Jiake $\mathrm{He}^{1,2}$, Wengen $\mathrm{Zhu}^{1}$, Yang $\mathrm{Yu}^{2}$, Jinzhu $\mathrm{Hu}^{1}$, Kui Hong ${ }^{1,3}$ \\ ${ }^{1}$ Department of Cardiovascular Medicine, The Second Affiliated \\ Hospital of Nanchang University, Nanchang, Jiangxi, China \\ ${ }^{2}$ Department of Pharmacy, The Second Affiliated Hospital of Nanchang University, Nanchang, Jiangxi, China \\ ${ }^{3}$ Jiangxi Key Laboratory of Molecular Medicine, The Second Affiliated \\ Hospital of Nanchang University, Nanchang, Jiangxi, China
}

\begin{abstract}
Background: Common genetic polymorphisms at chromosome $4 q 25$ were associated with increased susceptibility to atrial fibrillation $(A F)$. However, it remained controversial whether these variants could be used as risk predictors for AF recurrence after catheter ablation. We therefore performed a metaanalysis to quantify the association between rs2200733 $C>T / r s 10033464 G>T$ and $A F$ recurrence.

Methods: Relevant studies were systematically retrieved from PubMed, Web of Science, Elsevier database and Cochrane library through November 2016. Data were abstracted and pooled using Stata 12.0 software.

Results: A total of 2,145 patients undergoing catheter ablation were included. Patients with rs2200733 $T T$ or $T T+C T$ showed an overall increased susceptibility to AF recurrence (homozygous model [TT vs. CC]: odds ratio $[O R]=2.03,95 \%$ confidence interval $[C I] 1.49-2.76, p=0.000 ;$ dominant model $[T T+T C$ vs. $C C]:$ OR $=1.48,95 \%$ CI 1.17-1.87, $p=0.001$; recessive model $[T T$ vs. $T C+C C]:$ $O R=1.88,95 \% C I 1.12-3.15, p=0.017)$. Subgroup analysis also identified a positive relation in Caucasians and late recurrence of $A F$ in allelic, homozygous and dominant comparison. Moreover, a significant increased risk of AF recurrence was observed in patients with rs $10033464 T G$ or TT $+T G$ (heterozygous model $[T G$ vs. GG]: $O R=1.46,95 \%$ CI 1.01-2.12, $p=0.047$; dominant model $[T T+T G$ vs. $G G]:$ OR $=1.51,95 \%$ CI 1.04-2.17, $p=0.029)$.

Conclusions: After catheter ablation, rs2200733 (TT or TT+TC) and rs10033464 (TT+TG or TG) were associated with increased risk of AF recurrence. (Cardiol J 2018; 25, 5: 628-638)

Key words: atrial fibrillation, catheter ablation, rs2200733, rs10033464, meta-analysis
\end{abstract}

\section{Introduction}

Atrial fibrillation (AF) is one of the most prevalent forms of cardiac dysrhythmia in clinical practice, which affects 33.5 million individuals globally [1]. Several factors already have been identified to increase the susceptibility to AF, such as smoking, sex, and obesity [2-4]. Interestingly, genetic predisposition has been added into the long list of risk factors for AF. Gudbjartsson et al. [5] found common genetic polymorphisms at chromosome 4q25 (PITX2) locus, including rs2200733 C > T

Address for correspondence: Prof. Dr. Kui Hong, Department of Cardiovascular Medicine, Key Laboratory of Molecular Medicine of Jiangxi, The Second Affiliated Hospital of Nanchang University, Minde Road No. 1, Nanchang, Jiangxi, tel: +86-791-86312917, e-mail: hongkui88@163.com 
(at-risk allele $\mathrm{T}$ ) and rs10033464 G > T (at-risk allele $\mathrm{T}$ ), were associated with increased susceptibility to AF [5]. The association between rs2200733 and $\mathrm{AF}$ was further replicated in Indian [6], Spanish [7], Danish [8], Italian [9] and Polish [10] as well as Chinese populations [11]. Three different genotypes, CC, CT and TT were identified in rs 2200733 with minor allele frequency 0.21 to 0.39 across different ethnic groups [12, 13]. The prevalence of $\mathrm{rs} 10033464 \mathrm{G}>\mathrm{T}$ was reported from $19 \%$ to $32 \%$ predisposed to $\mathrm{AF}$ recurrence after ablation $[14,15]$. Several genetic models, including allelic (T vs. C for rs2200733, T vs. G for rs10033464), heterozygous (TC vs. CC for rs2200733, TG vs. GG for rs10033464), homozygous (TT vs. CC for rs2200733, TT vs. GG for rs10033464), dominant (TT+TC vs. CC for rs2200733, TT+TG vs. GG for rs10033464), recessive (TT vs. TC + CC for rs2200733, TT vs. TG + GG for rs10033464) models were applied to quantify the association between rs2200733/rs10033464 and AF recurrence after ablation $[10,15,16]$.

However, the relationship between two common polymorphisms (rs2200733 $\mathrm{C}>\mathrm{T}$ and rs $10033464 \mathrm{G}>\mathrm{T}$ ) at chromosome $4 \mathrm{q} 25$ locus and $\mathrm{AF}$ recurrence after catheter ablation remained controversial. Husser et al. [15] provided evidence that the presence of any variant allele (rs2200733 T or rs10033464 T) increased the risk of AF recurrence within the first 7 days and between 3 and 6 months in Caucasian patients of German descent. And both variants independently predicted AF recurrence when evaluated by dominant models in multivariable analysis [15]. In Chinese Han population, single nucleotide polymorphism (SNP) rs2200733 was an independent factor for AF recurrence after ablation, and the risk allele $\mathrm{T}$ was associated with AF recurrence [17]. Zhao et al. [16] also reported rs2200733 TT was able to predict a 1.8-fold increased risk for clinical recurrence after catheter ablation. On the contrary, Kiliszek et al. [18] found that rs2200733 C > T or rs 10033464 $\mathrm{G}>\mathrm{T}$ failed to correlate with $\mathrm{AF}$ recurrence after a single AF ablation in long-term follow-up (median of 45 months). In Korean patients, rs $2200733 \mathrm{~T}$ allele was strongly associated with $\mathrm{AF}$, but this SNP again failed to predict clinical recurrence after catheter ablation [12]. Therefore, the present study performed a meta-analysis to investigate the effect of common non-coding variants on chromosome 4q25 (rs2200733 C>T and rs10033464 $\mathrm{G}>\mathrm{T}$ ) on the risk of AF recurrence after catheter ablation.

\section{Methods}

\section{Literature search}

A systematic search of PubMed, Web of Science, Elsevier database and Cochrane library was undertaken for studies through November 2016. The search terms included all possible combinations "rs2200733”, "rs10033464”, “SNP”, “polymorphism or variant", "atrial fibrillation", "AF", "4q25 (PITX2)", "ablation", "outcome”, and "AF recurrence after ablation". Manual searches of study references were also conducted. Moreover, several related articles from reviews and other pertinent sources such as research bibliographies were inspected as well.

\section{Inclusion and exclusion criteria}

An initial screening for titles and abstracts was performed. A second screening was based on full-text review. The following criteria were used to evaluate whether a study was eligible:

a) The association between $\mathrm{rs} 2200733 / \mathrm{rs} 10033$ 464 and $\mathrm{AF}$ recurrence after ablation must be assessable.

b) The genotype data of rs2200733 and rs10033464 must be provided.

c) The study has a cohort design or has a casecontrol.

d) The research should report an estimate of risk for AF recurrence after ablation with a corresponding $95 \%$ confidence interval $(\mathrm{CI})$.

e) If the same population was studied in more than one study, study with larger sample size and more comprehensive outcome were included.

f) $\mathrm{AF}$ recurrence was defined according to the Heart Rhythm Society/European Heart Rhythm Association/European Cardiac Arrhythmia Society Consensus Statement recommendations. Late recurrence of AF (LRAF) was defined as any episode of atrial tachyarrhythmia, including atrial tachycardia, atrial flutter, or AF (AT/AF) lasting more than $30 \mathrm{~s}$ that occurred after a 3-month post-ablation blanking period. Early recurrence of AF (ERAF) was defined as an AF episode within 3 months post-ablation blanking period.

Studies were excluded if they met the following criteria:

a) It is a review, letter, conference abstracts, and/ /or case report.

b) The study lacked information on the risk of AF recurrence after ablation.

c) The study did not provide sufficient data. 


\section{Data extraction and quality assessment}

An outcome of interest in this study was the capability of rs2200733 and rs 10033464 to predict the risk of $\mathrm{AF}$ recurrence after catheter ablation. After careful review, data was extracted using a standardized data-collection form for each eligible article: first author's name, publication year, ethnicity/nationality, patient number, patient characteristics. Clinical end point, quality assessment, comorbid conditions, anatomic factors, ablation strategies, methods of AF recurrence ascertainment, and adjunctive post-operation therapy were also considered. The quality of each study was evaluated according to the Newcastle-Ottawa quality assessment scale (NOS) [19].

\section{Statistical analyses}

All statistical analyses were performed with Stata 12.0 software (StataCorp, College Station, TX, USA). Odds ratio (OR) was used as a common measure of the association between rs2200733 $\mathrm{C}>\mathrm{T} / \mathrm{rs} 10033464 \mathrm{G}>\mathrm{T}$ and $\mathrm{AF}$ recurrence. Pooled ORs were performed for allelic comparison ( $\mathrm{T}$ vs. C for rs2200733, T vs. G for rs10033464), heterozygote model (TC vs. CC for rs2200733, TG vs. GG for rs10033464), homozygote model (TT vs. CC for rs2200733, TT vs. GG for rs10033464), dominant model (TT+CT vs. CC for rs2200733, TT+GT vs. GG for rs10033464), recessive model (TT vs. TC $+\mathrm{CC}$ for rs2200733, TT vs. TG+GG for rs10033464), respectively. Cochran's Q test and $\mathrm{I}^{2}$ statistic were used to evaluate the consistency across studies. Fixed effect model was used when $\mathrm{p}<0.10$ and $\mathrm{I}^{2}<50 \%$; otherwise random effect model was used. Sensitivity analysis was performed through omitting individual study one-by-one to investigate the influence of single study on the overall risk estimates. Subgroup analysis was also performed where appropriate. Potential publication bias was checked by Begg's funnel plots and Egger's regression test. An asymmetric plot and $p$ value of the Egger test less than 0.05 was considered a significant publication bias. Unless otherwise stated, $\mathrm{p}<0.05$ was considered statistically significant.

\section{Results}

\section{Study characteristics}

A total of 32 records were identified through the above mentioned literature search strategy. After screening titles and abstracts in the first round, 12 articles were excluded. Following this, the remaining 20 articles, 7 articles were excluded that did not involve AF recurrence, rs2200733 or rs10033464. After reviewing full texts and data, 8 articles were excluded ( 5 articles without sufficient information on risk estimate, and 3 articles without ablation). Finally, 5 articles were eligible for inclusion in this study. Husser et al. [15] reported ORs for both ERAF and LRAF; each OR was included in this analysis. A total of 2,145 patients who underwent catheter ablation were included. The characteristics of the included studies were presented in Table 1 and Table 2 . The NOS scores of included studies were all more than 7 scores (high quality).

In general, $\mathrm{AF}$ ablation strategy was comparable among included studies. A 3-dimensional mapping system was used for catheter orientation, computed tomographic image integration, and tagging of the ablation sites (NavX /NavX-Ensite system [12, 15]; or CARTO system [16, 17]; or LocaLisa system [18]). Transseptal access was performed. And an irrigatedtip ablation catheter was used in 4 studies [12,15-17]. A non-irrigated catheter was used in 1 study [18] under the consideration of non-significant difference in $\mathrm{AF}$ recurrence between patients treated with irrigated and non-irrigated catheters [20]. Circumferential pulmonary vein isolation with bidirectional block was a major ablation endpoint at all centers. Additional ablation to the left atrial (LA) roof, basal posterior wall, posterior inferior wall, and anterior wall, mitral isthmus, cavotricuspid isthmus, superior vena cava, non-pulmonary vein (PV) foci, complex fractionated atrial electrograms were performed based on operator discretion.

\section{Genotypic association between rs2200733 $\mathrm{C}>\mathrm{T}$ and $\mathrm{AF}$ recurrence}

In the primary analysis, the associations between rs2200733 $\mathrm{C}>\mathrm{T}$ and $\mathrm{AF}$ recurrence after ablation were analyzed under five genetic models (Table 3). Fixed-effects model was used in the heterozygous, homozygous and dominant model. Random-effects model was used in the allelic and recessive model as a result of heterogeneity. Overall, significant positive relation was observed in four genetic models ( $\mathrm{T}$ vs. $\mathrm{C}$ : $\mathrm{OR}=1.50,95 \% \mathrm{CI}$ $1.18-1.90, \mathrm{p}=0.001$; TT vs. $\mathrm{CC}: \mathrm{OR}=2.03,95 \% \mathrm{CI}$ 1.49-2.76, $\mathrm{p}=0.000$; TT $+\mathrm{CT}$ vs. $\mathrm{CC}: \mathrm{OR}=1.48$, 95\% CI 1.17-1.87, $\mathrm{p}=0.001$; TT vs. TC $+\mathrm{CC}$ : $\mathrm{OR}=1.88,95 \%$ CI 1.12-3.15, $\mathrm{p}=0.017$; Fig. 1 ). No significant association was found in heterozygous comparison (TC vs. CC: $\mathrm{OR}=1.24,95 \% \mathrm{CI}$ $0.96-1.59, \mathrm{p}=0.096)$, but a trend of increased susceptibility still existed.

Subgroup analysis was conducted according to ethnicity and time to AF recurrence (Table 3). In Caucasians, a significant association was 

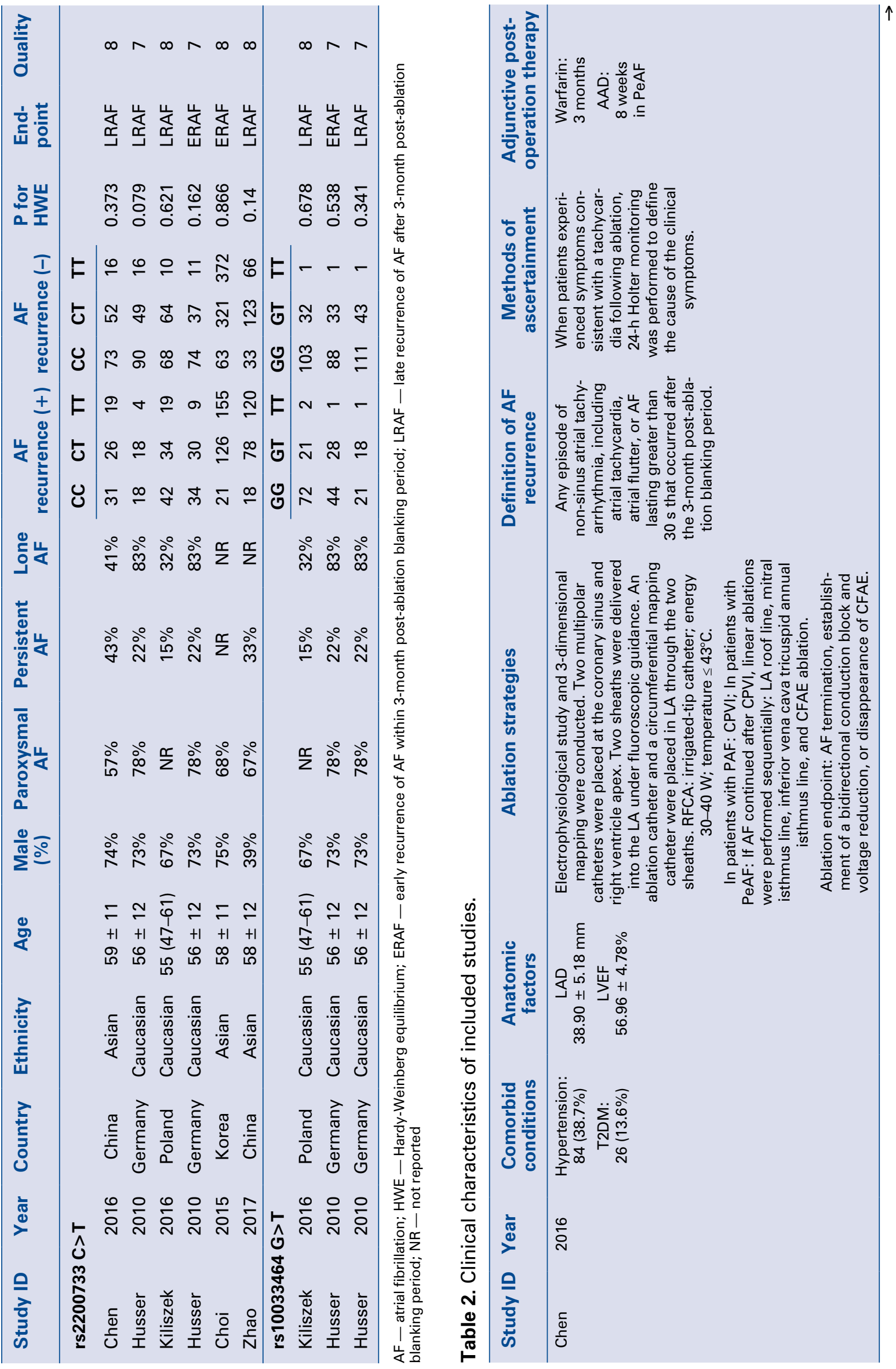


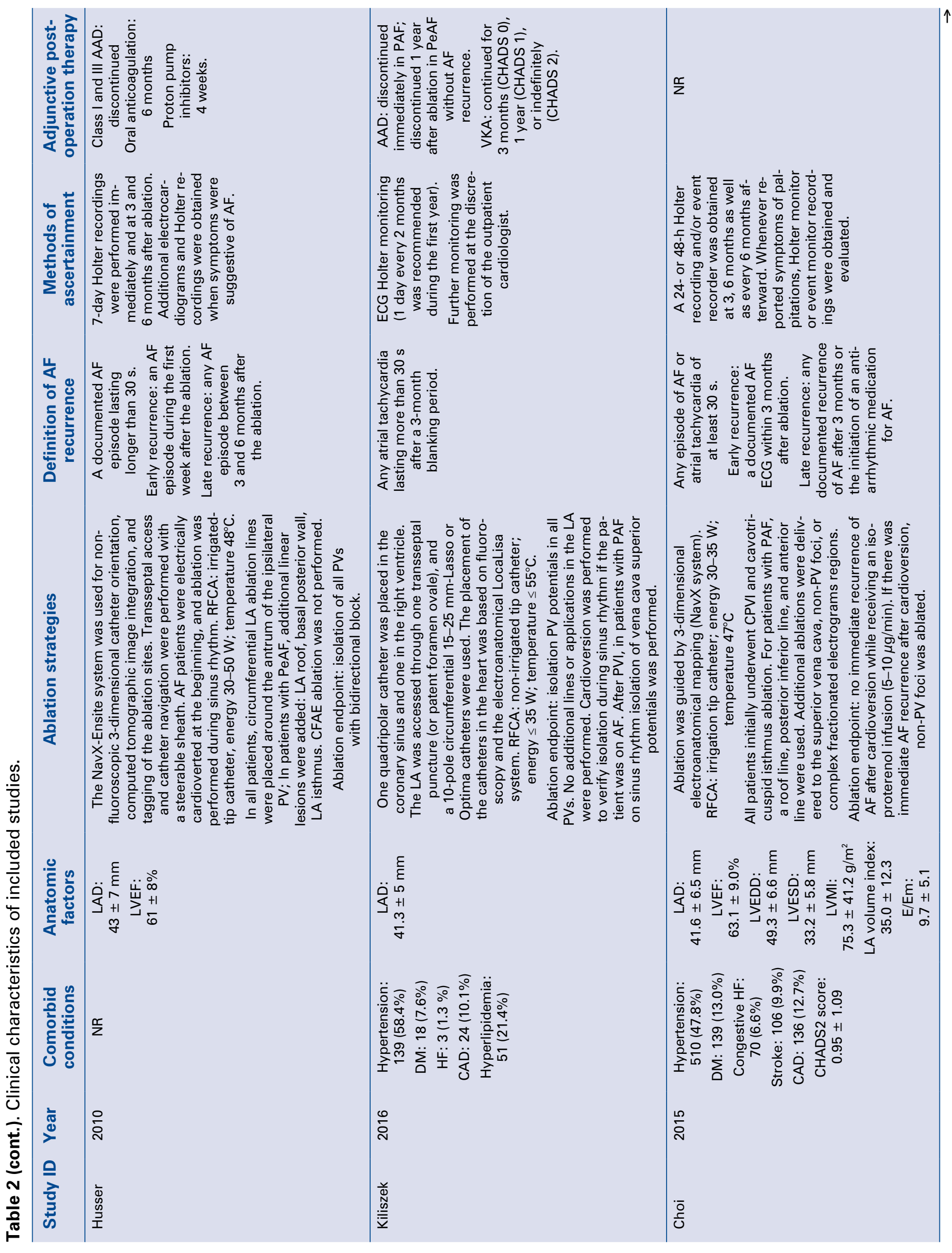



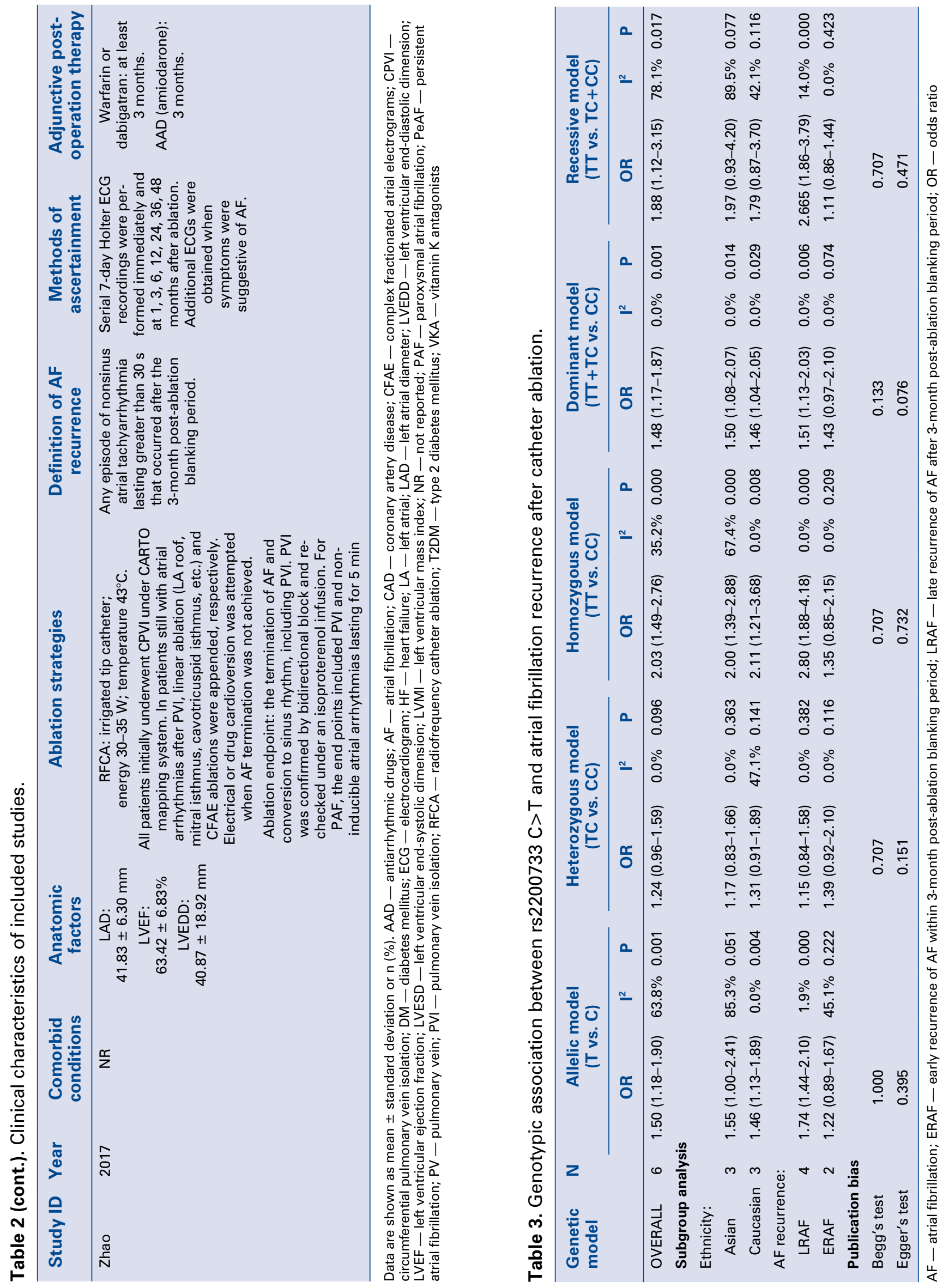


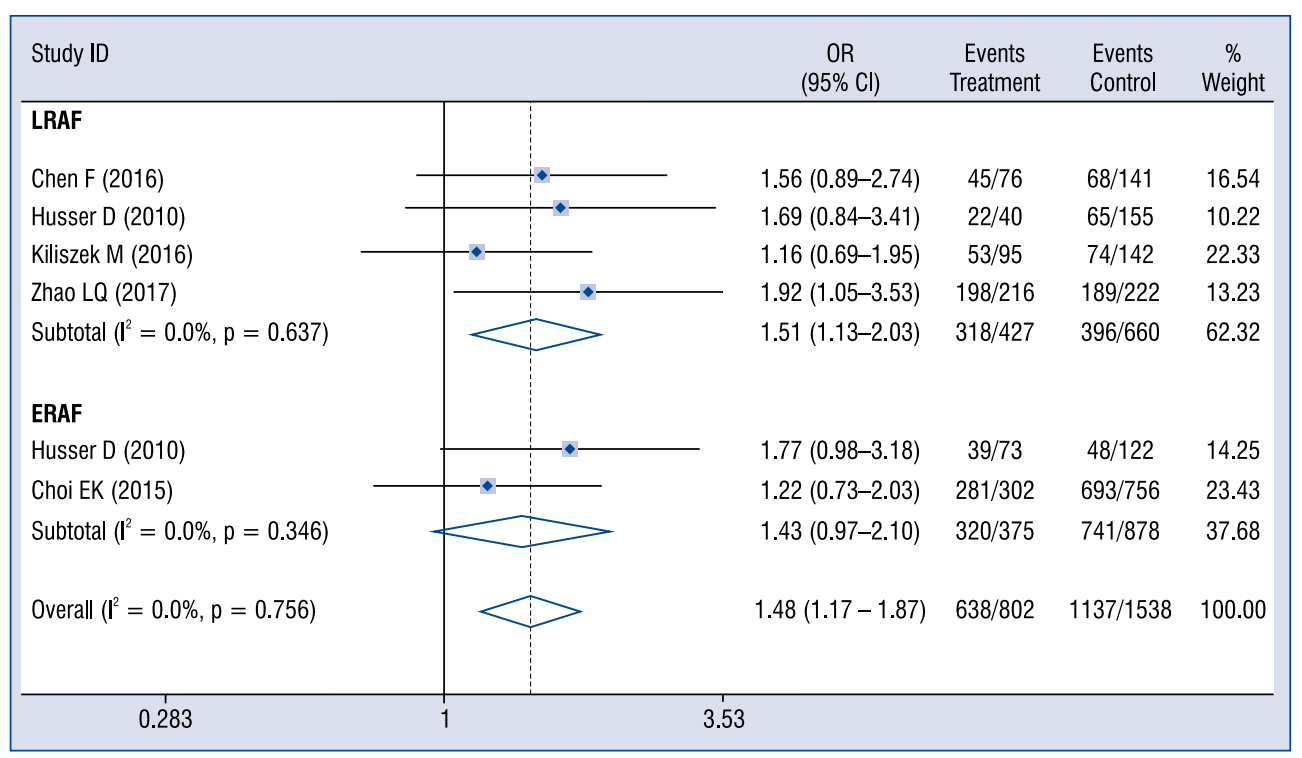

Figure 1. Forest plot of dominant model of rs2200733 for overall comparison (TT+CT vs. CC); LRAF — late recurrence of atrial fibrillation; ERAF - early recurrence of atrial fibrillation. $\mathrm{Cl}$ - confidence interval; OR - odds ratio.

observed in three genetic models except for the heterozygous model (TC vs. CC: $\mathrm{OR}=1.31,95 \%$ CI $0.91-1.89, \mathrm{p}=0.141$ ) and recessive model (TT vs. $\mathrm{TC}+\mathrm{CC}: \mathrm{OR}=1.79,95 \%$ CI $0.87-3.70, \mathrm{p}=$ $=0.116)$. A significant association was observed for LRAF in four genetic models except for heterozygous comparison (TC vs. $\mathrm{CC}$ : $\mathrm{OR}=1.15,95 \% \mathrm{CI}$ $0.84-1.58, \mathrm{p}=0.382)$. No association was found to be significant in any genetic models for ERAF.

\section{Genotypic association between rs10033464 $\mathrm{G}>\mathrm{T}$ and $\mathrm{AF}$ recurrence}

In the secondary analysis, the associations between rs $10033464 \mathrm{G}>\mathrm{T}$ and the risk of $\mathrm{AF}$ recurrence were evaluated. A significant increased risk of AF recurrence was identified in the allelic model ( $\mathrm{T}$ vs. $\mathrm{G}$ : $\mathrm{OR}=1.44,95 \%$ CI $1.04-2.00$, $\mathrm{p}=0.027$ ), heterozygous model (TG vs. GG: $\mathrm{OR}=1.46,95 \% \mathrm{CI} 1.01-2.12, \mathrm{p}=0.047)$ and dominant model (TT+TG vs. GG: OR $=1.51$, 95\% CI 1.04-2.17, $\mathrm{p}=0.029$, Table 4, Fig. 2). And a non-significant association was observed in homozygous comparison (TT vs. GG) or recessive comparison (TT vs. TG+GG). However, this result should be interpreted with caution due to a limited number of studies.

\section{Sensitivity analysis and publication bias}

Sensitivity analysis was performed to evaluate the influence of single study on the pooled ORs for rs2200733 C > T and rs10033464 G > T by deleting each study once in every genetic model. Consistently, the pooled estimate remained non- significant. No publication bias for the association between rs2200733 $\mathrm{C}>\mathrm{T}$ and $\mathrm{AF}$ susceptibility was identified by Begg's funnel plot $(\mathrm{p}=0.133$; Table 3) or Egger's regression test $(\mathrm{p}=0.075$; Table 3). Symmetrical funnel plots were obtained in all five genetic models. Due to limited studies, no funnel plot or Egger tests were performed for the association between $\mathrm{rs} 10033464 \mathrm{G}>\mathrm{T}$ and the risk of $\mathrm{AF}$ recurrence after ablation.

\section{Discussion}

Catheter ablation has been employed as a first-line treatment for rhythm control in many patients with $\mathrm{AF}$, especially in whom failed in anti-arrhythmic drug treatment [21]. Although ablation is effective [22], large effective variances ranging from $29 \%$ to $90 \%$ in ablation outcome are observed in real clinical setting [21,23]. Winkle et al. [24] established a clinical scoring system to predict long-term freedom from $\mathrm{AF}$ after ablation. Genome-wide association studies identified several AF-susceptibility loci, such as rs2200733 C > T and rs10033464 $\mathrm{G}>\mathrm{T}$ at chromosome 4q25, could be used as prognostic factors. And recent studies have focused on their potential to predict $\mathrm{AF}$ risk in patients undergoing ablation.

In this meta-analysis, carriers of at least 1 risk allele conferred an increased risk of $48 \%$ or $51 \%$ for $\mathrm{AF}$ recurrence when evaluated by dominant model for rs 2200733 or rs 10033464 , respectively. However, the mechanisms underlying these associations remained elusive. These noncoding 


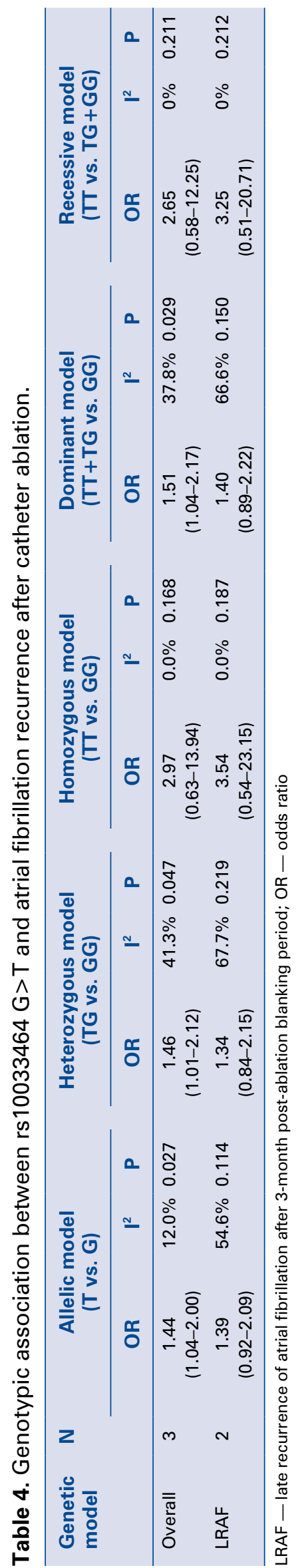

variants existed in close proximity $(\sim 150 \mathrm{~kb})$ to the cis-regulatory region of paired-like homeodomain transcription factor 2 (PITX2), and modulated PITX2 activity through transcription level as postulated [25, 26]. PITX2 efficiently regulated atrial resting membrane potential and involved in the formation, differentiation and proliferation of pulmonary myocardium $[27,28]$. Three isoforms of PITX2 (PITX2a, PITX2b, and PITX2c) have been identified, among which PITX2c was the only isoform expressed in the left atrium [29]. PITX2c directs formation of left and right anatomic characteristics and mediates left-right asymmetry signaling cascade $[28,30]$. It also efficiently modulates ion channels and calcium handling proteins in atrial cardiomyocytes [28, 31,32]. Down-regulation or up-regulation of PITX2c is associated with $\mathrm{AF}$ arrhythmogenesis [31, 32]. In human patients with sustained AF, PITX2c insufficiency could lead to atrial electrical and structural remodeling $[10,17$, 33]. Taking these findings together, we speculated that rs2200733 or rs10033464 could modulate the expression of PITX2c. And PITX2c-mediated alterations in PV phenotype (such as arrhythmogenicity of PV myocardium, PV myocardial sleeve or LA/PV electrophysiology) affected response to $\mathrm{PV}$ isolation (PVI). However, PVI itself might be different between the centers: size of isolation area around PV, durability of PVI lesion or the usage of the contact force sensing ablation catheter etc. Thus further prospective researches, especially large-scale multicenter trials with standardized clinical procedures, are required to solve these issues.

In accordance with the finding of Husser et al. [15], a significant increased risk of LRAF was identified in rs10033464 in allelic, heterozygous and dominant comparison, but such association needs to be further validated as a result of limited studies. We also observed a robust genotypic association between rs2200733 and LRAF in allelic model, homozygous model, dominant model and recessive genetic model, which were consistent with precious reports of Husser et al. [15], Zhao et al. [16], Chen et al. [17] and Benjamin Shoemaker et al. [34]. But no association was found to be significant in any genetic models for ERAF. Choi et al. [12] reported lack of association between rs2200733 and LRAF. Kiliszek et al. [18] found rs2200733 C>T or rs10033464 G>T failed to correlate with AF recurrence in a median of 45 months follow-up. Many potential causes could underlie the discordant results. The incidences of recurrence in the entire follow-up period were 


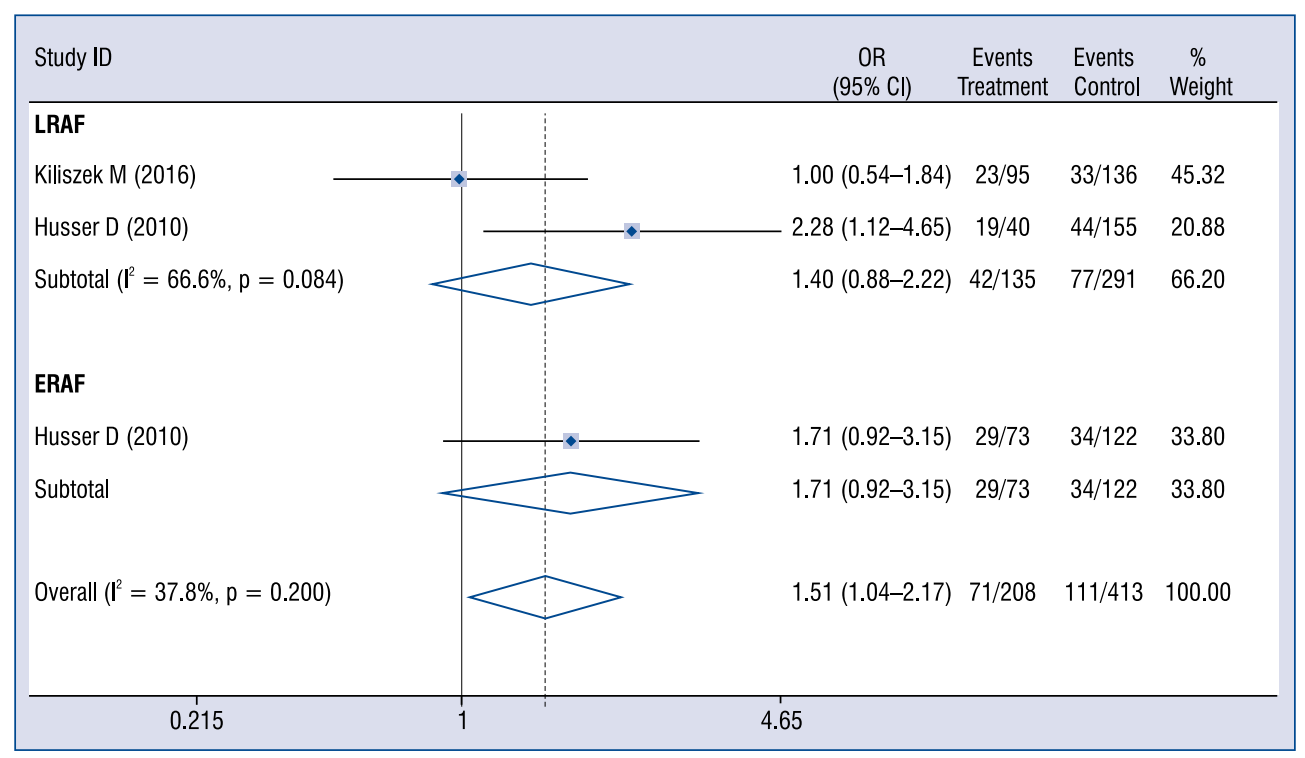

Figure 2. Forest plot of dominant model of rs10033464 for overall comparison (TT+GT vs. GG); LRAF - late recurrence of atrial fibrillation; ERAF — early recurrence of atrial fibrillation. $\mathrm{Cl}$ - confidence interval; OR - odds ratio.

not provided in Choi's and Kiliszek's studies, thus data for ERAF in Korean and 6-month AF recurrence in Polish were used in the current meta-analysis. It is possible that the influence of genetic factors might have different impart on AF recurrence depending on post-ablation period (occurring within 3-months or later than 3-months after ablation). Other potential causes, such as the variability of technical approach to ablation procedure, ability for AF surveillance, frequency of polymorphism among studied populations, composition of AF patients and sample size, as well as the adjusted covariates, would be confounders in $\mathrm{AF}$ recurrence.

\section{Conclusions}

Common 4q25 genetic variants rs2200733 (TT or $\mathrm{TT}+\mathrm{TC})$ and rs10033464 (TT+TG or TG) are associated with increased risk of $\mathrm{AF}$ recurrence. And rs2200733 C > T can be used as a clinical marker for outcome prediction before embarking on the procedure. Further prospective studies, especially large-scale multicenter trials with standardized clinical procedures, are warranted to ascertain the mechanistic relationship between rs2200733/rs10033464 and clinical response after ablation. These studies will eventually contribute to establishing the genotype-based algorithm for prediction and developing the genotype-guided therapy in clinical practice.

\section{Limitations of the study}

Under review was the effect of rs2200733 and rs 10033464 on the risk of AF recurrence after catheter ablation, but the following limitations are still worth mentioning. First, this is a retrospective observational study that needs prospective confirmation. Unavoidable differences and some variability introduced by operators exist between centers, which may diminish the strength of observed associations or contribute to the lack of an association in heterozygous comparison or recessive comparison in subgroup analysis. Second, it is possible that variability and accuracy together with the intensity of AF monitoring after ablation inherently limited the interpretation of data in the current study. Asymptomatic AF after ablation was not investigated in all centers. Third, the presence of 1 variant indicated an increased risk for $\mathrm{AF}$ recurrence, but variant carriers vary greatly in different centers and represent only a minority of the AF population. Thus, prospective studies, especially large-scale multicenter trials with standardized clinical procedures are warranted to fully appreciate their risk. Fourth, although it was speculated that these variants modulate the expression of PITX2c and result in alterations in PV phenotype, PV-LA reconduction is still the prevailing cause for AF recurrence after ablation. Other mechanisms such as progression of low voltage area in LA, non-PV foci also may be associated with $\mathrm{AF}$ recidivism. However, because whether 
re-ablations were performed or not has remained unknown during the follow-up period in these centers, reconduction could not be assessed. AF recurrences might be due to different mechanisms that are not captured with the analyzed variants in these studies. And it is also worth noting that $4 \mathrm{q} 25$ variants may simply be used as one of the clinical markers for outcome prediction. Finally, given the fact that PITX2 adjacent non-coding RNA and enhancer binding protein 2 alpha were involved in regulation of PITX2c expression [35, 36], it cannot be ruled out that rs2200733 or rs10033464 variants may also control the expression of coding or non-coding regions of other AF-associated genes or SNPs through gene-gene interaction [37]. Not all AF-susceptibility variants which were in linkage disequilibrium with these variants were included in this analysis. The impact of potential gene-gene interaction at $4 \mathrm{q} 25$ locus to the clinical response for ablation remained unknown.

\section{Acknowledgements}

This work was supported by the National Basic Research Program of China (973 program 2013CB31103), the National Nature Science Foundation of China $(8153000545,81603188)$, the China Postdoctoral Science Foundation (2017M612165), the grant from Jiangxi Scientific Program (20151BBB70266, 20151BAB215041).

\section{Conflict of interest: None declared}

\section{References}

1. Chugh SS, Havmoeller R, Narayanan K, et al. Worldwide epidemiology of atrial fibrillation: a Global Burden of Disease 2010 Study. Circulation. 2014; 129(8): 837-847, doi: 10.1161/CIRCULATIONAHA.113.005119, indexed in Pubmed: 24345399.

2. Zhu W, Yuan P, Shen Y, et al. Association of smoking with the risk of incident atrial fibrillation: A meta-analysis of prospective studies. Int J Cardiol. 2016; 218: 259-266, doi: 10.1016/j. ijcard.2016.05.013, indexed in Pubmed: 27236125.

3. Piccini JP, Simon DN, Steinberg BA, et al. Differences in Clinical and Functional Outcomes of Atrial Fibrillation in Women and Men: Two-Year Results From the ORBIT-AF Registry. JAMA Cardiol. 2016; 1(3): 282-291, doi: 10.1001/jamacardio.2016.0529, indexed in Pubmed: 27438106.

4. Phan K, Khuong JN, Xu J, et al. Obesity and postoperative atrial fibrillation in patients undergoing cardiac surgery: Systematic review and meta-analysis. Int J Cardiol. 2016; 217: 49-57, doi: 10.1016/j.ijcard.2016.05.002, indexed in Pubmed: 27179208.

5. Gudbjartsson DF, Arnar DO, Helgadottir A, et al. Variants conferring risk of atrial fibrillation on chromosome 4q25. Nature. 2007; 448(7151): 353-357, doi: 10.1038/nature06007, indexed in Pubmed: 17603472.

6. Bhanushali A, Nair A, Jagdale G, et al. Association of Genetic Variants at the $4 \mathrm{q} 25$ Locus with Atrial Fibrillation in Indian
Population. J Clin Lab Anal. 2017; 31(1), doi: 10.1002/jcla.22017, indexed in Pubmed: 27346453.

7. Ferrán A, Alegret JM, Subirana I, et al. Association between rs2200733 and rs7193343 genetic variants and atrial fibrillation in a Spanish population, and meta-analysis of previous studies. Rev Esp Cardiol (Engl Ed). 2014; 67(10): 822-829, doi: 10.1016/j. rec.2013.12.019, indexed in Pubmed: 25262128.

8. Olesen MS, Holst AG, Jabbari J, et al. Genetic loci on chromosomes $4 \mathrm{q} 25,7 \mathrm{p} 31$, and $12 \mathrm{p} 12$ are associated with onset of lone atrial fibrillation before the age of 40 years. Can J Cardiol. 2012; 28(2): 191-195, doi: 10.1016/j.cjca.2011.11.016, indexed in Pubmed: 22336519 .

9. Viviani Anselmi C, Novelli V, Roncarati R, et al. Association of rs2200733 at 4q25 with atrial flutter/fibrillation diseases in an Italian population. Heart. 2008; 94(11): 1394-1396, doi: 10.1136/ hrt.2008.148544, indexed in Pubmed: 18931155.

10. Kiliszek M, Franaszczyk M, Kozluk E, et al. Association between variants on chromosome 4q25, 16q22 and 1q21 and atrial fibrillation in the Polish population. PLoS One. 2011; 6(7): e21790, doi: 10.1371/journal.pone.0021790, indexed in Pubmed: 21760908.

11. Lee KT, Yeh HY, Tung CP, et al. Association of RS2200733 but not RS10033464 on 4q25 with atrial fibrillation based on the recessive model in a Taiwanese population. Cardiology. 2010; 116(3): 151-156, doi: 10.1159/000318172, indexed in Pubmed: 20606429 .

12. Choi EK, Park JH, Lee JY, et al. Korean Atrial Fibrillation (AF) Network: Genetic Variants for AF Do Not Predict Ablation Success. J Am Heart Assoc. 2015; 4(8): e002046, doi: 10.1161/ JAHA.115.002046, indexed in Pubmed: 26272656.

13. Lubitz SA, Sinner MF, Lunetta KL, et al. Independent susceptibility markers for atrial fibrillation on chromosome 4q25. Circulation. 2010; 122(10): 976-984, doi: 10.1161/CIRCULATIONAHA.109.886440, indexed in Pubmed: 20733104.

14. Kääb S, Darbar D, van Noord C, et al. Large scale replication and meta-analysis of variants on chromosome 4q25 associated with atrial fibrillation. Eur Heart J. 2009; 30(7): 813-819, doi: 10.1093/ eurheartj/ehn578, indexed in Pubmed: 19141561.

15. Husser D, Adams V, Piorkowski C, et al. Chromosome 4q25 variants and atrial fibrillation recurrence after catheter ablation. J Am Coll Cardiol. 2010; 55(8): 747-753, doi: 10.1016/j. jacc.2009.11.041, indexed in Pubmed: 20170812.

16. Zhao LQ, Zhang GB, Wen ZJ, et al. Common variants predict recurrence after nonfamilial atrial fibrillation ablation in Chinese Han population. Int J Cardiol. 2017; 227: 360-366, doi: 10.1016/j. ijcard.2016.11.057, indexed in Pubmed: 27843048.

17. Chen F, Yang Y, Zhang R, et al. Polymorphism rs2200733 at chromosome $4 \mathrm{q} 25$ is associated with atrial fibrillation recurrence after radiofrequency catheter ablation in the Chinese Han population. Am J Transl Res. 2016; 8(2): 688-697, indexed in Pubmed: 27158361.

18. Kiliszek M, Kozluk E, Franaszczyk M, et al. The 4q25, 1q21, and 16q22 polymorphisms and recurrence of atrial fibrillation after pulmonary vein isolation. Arch Med Sci. 2016; 12(1): 38-44, doi: 10.5114/aoms.2015.48284, indexed in Pubmed: 26925117.

19. Stang A. Critical evaluation of the Newcastle-Ottawa scale for the assessment of the quality of nonrandomized studies in metaanalyses. Eur J Epidemiol. 2010; 25(9): 603-605, doi: 10.1007/ s10654-010-9491-z, indexed in Pubmed: 20652370.

20. Cappato R, Calkins H, Chen SA, et al. Updated worldwide survey on the methods, efficacy, and safety of catheter ablation for 
human atrial fibrillation. Circ Arrhythm Electrophysiol. 2010; 3(1): 32-38, doi: 10.1161/CIRCEP.109.859116, indexed in Pubmed: 19995881.

21. Kirchhof P, Calkins H. Catheter ablation in patients with persistent atrial fibrillation. Eur Heart J. 2017; 38(1): 20-26, doi: 10.1093/eurheartj/ehw260, indexed in Pubmed: 27389907.

22. Björkenheim A, Brandes A, Chemnitz A, et al. Rhythm control and its relation to symptoms during the first two years after radiofrequency ablation for atrial fibrillation. Pacing Clin Electrophysiol. 2016; 39(9): 914-925, doi: 10.1111/pace.12916, indexed in Pubmed: 27418324.

23. Tzou WS, Marchlinski FE, Zado ES, et al. Long-term outcome after successful catheter ablation of atrial fibrillation. Circ Arrhythm Electrophysiol. 2010; 3(3): 237-242, doi: 10.1161/CIRCEP.109.923771, indexed in Pubmed: 20335557.

24. Winkle RA, Jarman JWE, Mead RH, et al. Predicting atrial fibrillation ablation outcome: The CAAP-AF score. Heart Rhythm. 2016; 13(11): 2119-2125, doi: 10.1016/j.hrthm.2016.07.018, indexed in Pubmed: 27435586.

25. Li N, Dobrev D, Wehrens XHT. PITX2: a master regulator of cardiac channelopathy in atrial fibrillation? Cardiovasc Res. 2016; 109(3): 345-347, doi: 10.1093/cvr/cvw008, indexed in Pubmed: 26782118.

26. Shoemaker MB, Bollmann A, Lubitz SA, et al. Common genetic variants and response to atrial fibrillation ablation. Circ Arrhythm Electrophysiol. 2015; 8(2): 296-302, doi: 10.1161/CIRCEP.114.001909, indexed in Pubmed: 25684755.

27. Mommersteeg MTM, Brown NA, Prall OWJ, et al. Pitx2c and Nkx2-5 are required for the formation and identity of the pulmonary myocardium. Circ Res. 2007; 101(9): 902-909, doi: 10.1161/ CIRCRESAHA.107.161182, indexed in Pubmed: 17823370.

28. Syeda F, Holmes AP, Yu TY, et al. PITX2 modulates atrial membrane potential and the antiarrhythmic effects of sodiumchannel blockers. J Am Coll Cardiol. 2016; 68(17): 1881-1894, doi: 10.1016/j.jacc.2016.07.766, indexed in Pubmed: 27765191.

29. Kirchhof P, Kahr PC, Kaese S, et al. PITX2c is expressed in the adult left atrium, and reducing Pitx2c expression promotes atrial fibrillation inducibility and complex changes in gene expression.
Circ Cardiovasc Genet. 2011; 4(2): 123-133, doi: 10.1161/CIRCGENETICS.110.958058, indexed in Pubmed: 21282332.

30. Wang J, Klysik E, Sood S, et al. Pitx2 prevents susceptibility to atrial arrhythmias by inhibiting left-sided pacemaker specification. Proc Natl Acad Sci U S A. 2010; 107(21): 9753-9758, doi: 10.1073/pnas.0912585107, indexed in Pubmed: 20457925.

31. Lozano-Velasco E, Hernández-Torres F, Daimi H, et al. Pitx2 impairs calcium handling in a dose-dependent manner by modulating Wnt signalling. Cardiovasc Res. 2016; 109(1): 55-66, doi: 10.1093/cvr/cvv207, indexed in Pubmed: 26243430.

32. Pérez-Hernández M, Matamoros M, Barana A, et al. Pitx2c increases in atrial myocytes from chronic atrial fibrillation patients enhancing IKs and decreasing ICa,L. Cardiovasc Res. 2016; 109(3): 431-441, doi: 10.1093/cvr/cvv280, indexed in Pubmed: 26714926.

33. Chinchilla A, Daimi H, Lozano-Velasco E, et al. PITX2 insufficiency leads to atrial electrical and structural remodeling linked to arrhythmogenesis. Circ Cardiovasc Genet. 2011; 4(3): 269-279, doi: 10.1161/CIRCGENETICS.110.958116, indexed in Pubmed: 21511879.

34. Benjamin Shoemaker M, Muhammad R, Parvez B, et al. Common atrial fibrillation risk alleles at $4 \mathrm{q} 25$ predict recurrence after catheter-based atrial fibrillation ablation. Heart Rhythm. 2013; 10(3): 394-400, doi: 10.1016/j.hrthm.2012.11.012, indexed in Pubmed: 23178686.

35. Ye J, Tucker NR, Weng LC, et al. A Functional Variant Associated with Atrial Fibrillation Regulates PITX2c Expression through TFAP2a. Am J Hum Genet. 2016; 99(6): 1281-1291, doi: 10.1016/j.ajhg.2016.10.001, indexed in Pubmed: 27866707.

36. Gore-Panter SR, Hsu J, Barnard J, et al. PANCR, the PITX2 Adjacent Noncoding RNA, Is Expressed in Human Left Atria and Regulates PITX2c Expression. Circ Arrhythm Electrophysiol. 2016; 9(1): e003197, doi: 10.1161/CIRCEP.115.003197, indexed in Pubmed: 26783232.

37. Huang Y, Wang C, Yao Y, et al. Molecular Basis of Gene-Gene Interaction: Cyclic Cross-Regulation of Gene Expression and Post-GWAS Gene-Gene Interaction Involved in Atrial Fibrillation. PLoS Genet. 2015; 11(8): e1005393, doi: 10.1371/journal. pgen.1005393, indexed in Pubmed: 26267381. 